\title{
The Experience of Women in a Phase II Trial of Hypnosis and Progressive Muscle Relaxation for Body Image: Exploring Possible Mechanisms
}

Noel M Arring

University of Michigan School of Nursing

Carolyn K Lafferty ( $\square$ claffer2@utk.edu )

University of Michigan School of Nursing https://orcid.org/0000-0003-0849-3893

Patricia M Clark

University of Michigan School of Nursing

Debra L Barton

University of Michigan, School of Nursing

\section{Research Article}

Keywords: Body image, women's health, cancer, hypnosis, progressive muscle relaxation

Posted Date: June 15th, 2021

DOI: https://doi.org/10.21203/rs.3.rs-617453/v1

License: (c) (i) This work is licensed under a Creative Commons Attribution 4.0 International License.

Read Full License 


\section{Abstract}

\section{Purpose:}

The purpose of this study was to explore potential mechanisms of two interventions, hypnosis and progressive muscle relaxation to nature sounds (PMR), to improve body image in a randomized phase II trial. Participant comments were explored in an unplanned qualitative analysis.

\section{Methods:}

This qualitative study employed thematic analysis, a hybrid approach of inductive and deductive coding. We analyzed participant feedback given at any point during the 6-week intervention.

Results:

In the parent study 87 women were randomized in a 2:1 fashion, 59 to hypnosis and 28 to PMR. Sixtythree of the 87 women participating (72\%) were motivated to write comments elaborating on their study experience. Thematic analysis generated five themes, including: relaxation and stress management, mind-body connection, improved mood, sleep, and sexual health. Four of the themes were represented in both groups while sexual health was only represented in the hypnosis group.

\section{Conclusions:}

This analysis suggests that both hypnosis and PMR may improve body image through the ability to relax and manage stress, sleep better, improve mood and create a mind-body connection. Sexual health emerged as an important theme among participants in the hypnosis group, but not the PMR group. Hypnosis, due to the hypnotic suggestions for sexual health, may have a positive impact on sexual health specifically. Improving body image alone may not have profound effects on the broader concept of sexual health. More research is needed.

\section{Background And Significance}

There are over 4.6 million women with a history of breast or gynecological cancer in the United States [1], many of whom face long-term consequences from their cancer and/or cancer treatment. Sexual health is a key component of a healthy life [2]. One of the distressing long-term and often unaddressed consequences of breast and gynecological cancer and/or treatment is the negative impact on women's sexual health [3].

\section{Body Image: One Component of Sexual Health}

Female sexual health is a multi-component concept defined by the World Health Organization (WHO) as "a state of physical, emotional, mental, and social well-being in relation to sexuality, not merely the absence of disease, dysfunction or infirmity" [4]. Evidence demonstrates remarkable consistency around 
four key predictors of female sexual health: vaginal symptoms, partner concerns, sexual energy/libido, and body-image [5-9]. Body-image, the focus of the present study, can be defined as feelings, thoughts, and/or perceptions about one's body. Breast and gynecologic cancer survivors commonly report negative body image. For example, studies including measures of sexual function in breast cancer survivors demonstrate that up to $82 \%$ fall into the sexual dysfunction range with body satisfaction dropping below published norms [10]. In a recent qualitative study of gynecological cancer survivors, body image emerged as one of 15 factors impacting sexual relationships. Despite being among the most difficult challenges for survivors to adjust to following treatment, it was categorized as a modifiable factor, or one that may be influenced by the actions of a health-care provider [11].

\section{Mind-Body Connection}

Research examining mindfulness, cognitive behavioral therapy, and psycho-education interventions supports the potential usefulness of a mind-body approach to interventions targeting body image [9]. At the same time, literature in this area has limited scope, the benefits demonstrated are inconsistent or incomplete, and the intervention delivery methods generally required in-person group therapy over numerous sessions making them impractical and costly. Despite these limitations, the evidence is persuasive enough to consider novel mind-body interventions that could be effective as well as practical, easy to disseminate, and cost saving.

Investigators developed a feasibility study followed by a randomized pilot study to evaluate the effect of hypnosis as a mind-body therapy to improve body image. Hypnosis brings about a state of consciousness involving focused attention and reduced peripheral awareness characterized by a capacity for response to suggestion [12]. One hypothesis is that hypnotic suggestions and imagery techniques related to body image could be used to re-attain or attain a positive view of one's body and/or sexual self.

Based on a successful feasibility study which showed a large effect size for body image improvement [13], our research team conducted a randomized phase II trial evaluating hypnosis for body image in women with breast or gynecologic cancer. Progressive muscle relaxation (PMR) was chosen as the control arm to control for the effects of simple relaxation [13]. The primary outcome was the Impact of Treatment Scale (ITS), a measure that asks about body image change perception. It has been shown to discriminate between women with lower and higher sexual satisfaction and has demonstrated reliability and validity in women with gynecologic or breast cancer [10].

There were no statistically significant differences between the intervention (hypnosis) and control (PMR) groups on the ITS in the randomized phase II study. Both groups reported significant improvements in perception of body image (the primary outcome) from baseline to 6 weeks, had similar practice rates, and showed good adherence which did not appear to impact the outcomes. There were non-statistically significant differences between the groups in secondary outcomes with the hypnosis group reporting greater improvements than the PMR group in perceived sexuality and sexual satisfaction [13]. This incongruence in outcome impact is puzzling and in particular, the benefit from PMR was surprising. 
Therefore, in an exploratory, unplanned analysis, we used qualitative data provided by women in both arms of the study throughout the six week period to attempt to interrogate the relationship between body image and PMR and body image and hypnosis. Our aim was to determine whether mechanistic hypotheses could be developed for further testing.

\section{Methods}

The randomized phase II study methods and results have been reported in detail elsewhere [13] but will be described here briefly. The study was approved by the University of Michigan Medical School Institutional Review Board. Eighty-seven women were randomized in a 2:1 fashion, 59 to hypnosis (68\%) and 28 to PMR (32\%). Participants who were motivated to leave qualitative feedback were included in this secondary analysis.

\section{Eligibility}

Women were eligible to participate for the parent study if they met the following criteria: being diagnosed with breast or gynecologic cancer at any stage, undergoing or completed treatment, and reported a negative change in body image since diagnosis with a desire to improve that perception.

\section{Study Arms}

In each arm, participants attended 3 face-to-face sessions with a therapist trained in hypnosis and PMR. The sessions were spaced two weeks apart and spanned the six weeks of the study. For both groups, sessions lasted approximately 60 minutes, with about 20 minutes dedicated to engaging in the intervention, hypnosis or PMR. The PMR intervention focused on assessing sources of stress and teaching the basics of PMR practice. The hypnosis intervention focused on topics such as wellness, confidence and body image related to sexuality. Intervention sessions were recorded and transferred to a $C D$ for home practice throughout the week. While neither arm was described as the control group, the PMR arm was specifically not directed toward body image or sexual health, but rather on stress reduction and relaxation.

PMR participants were taught to tighten and then relax every muscle group, starting from the top of the head and continuing to the bottom of the feet. During sessions with the therapist, they worked on the process together. At home, the participant practiced the routine while listening to one of four audio recordings: ocean waves, a waterfall, a stream or white noise.

Hypnosis participants listened to the therapist who used a standardized script addressing feelings about the body and qualities that contribute to a sense of sexuality. For example, "you will be able to have positive feelings about your body, feeling whole and beautiful...you will experience more satisfaction in your sexual needs...satisfied with yourself as a sexual person... more emotional closeness when expressing yourself sexually ....and more aware of your sexual feelings when they occur...you will be 
pleased as these things occur both now and in the future." At home, the participant listened to a recording of the therapist directed session.

\section{Data Collection}

Specific open ended questions were not a required component of the study. However, opportunities to include comments were available at multiple points during the six-week intervention. The following paper and pen questionnaires left space for comments or had room in the margins where participants opted to supply information:

1) A daily practice log where participants recorded minutes practiced throughout the six-week intervention.

2) A side effect questionnaire completed at the end of each practice week and used to monitor for negative experiences with intervention. An open ended question was included that read "Did you experience any new emotions or physical effects this past week from being involved in the study treatment that we did not mention? If yes, please list."

3) A global impression of change scale, completed at the end of the study.

\section{Analysis}

Two members of the study team, both with research degrees and training in qualitative methods, extracted all comments from the three data sources listed above. Analysis generally followed a hybrid, six-stage process incorporating inductive and deductive thematic analysis: 1) develop a code manual; 2) test the reliability of the codes; 3 ) summarize data and identify preliminary themes; 4) apply template of codes and additional coding; 5) connect the codes and identifying themes; and 6) corroborate and legitimate coded themes [14]. The two reviewers independently coded the data. Deductive logic, or the use of a priori codes (relaxation/stress management, normalizing of the problem and mind-body connection), was introduced in stage four, resulting in the addition of three possible codes to the codebook.

\section{Results}

Of the 87 participants in the parent study, 63 women $(72 \%)$ were motivated to write comments elaborating on their study experience. Forty-four women in the hypnosis arm (75\%) wrote comments and 19 in the PMR group (68\%) wrote comments.

There were comments submitted in all of the areas available. Sixty-one women opted to leave feedback in the blank space labeled "comments on the daily practice log." Thirty-nine women offered comments in response to the question about other effects and 14 women wrote comments in the margins of the Global Impression of Change Scale. 
No differences were found between groups with respect to demographics [13]. Thematic analysis generated five themes, including: relaxation and stress management, sleep, mind-body connection, improved mood, and sexual health. Two of the apriori themes, relaxation/stress management and mindbody connection, were supported. Normalizing the problem was not supported by the comments. Three additional themes were identified: sleep, improved mood, and sexual health. Themes and supportive quotations, along with the point in time that they were collected, appear below.

\section{Relaxation and Stress Management}

The a priori code relaxation and stress management was evident as a theme in both study arms throughout the six week intervention. Clear statements attesting to the relaxation benefits of both arms began as early as week one and continued throughout the intervention (see Table 1).

\section{Sleep}

Sleep (not a priori) was a consistent theme in both study arms and supports the ability of both hypnosis and PMR to induce relaxation that is complete enough to, in some cases, induce sleep. There were many comments related to falling asleep during the intervention. Additionally, some participants made comments about getting better sleep in general since starting the intervention. Examples of both types of sleep-related comments can be found in Table 2.

\section{Mind-Body Connection}

A second a priori code that was supported by data related to a mind-body connection. Unlike relaxation quotes that either used some form of the word relax or focused on the participant's mental or emotional experience, mind-body connection quotations included some mention of the mental/emotional experience and the physical experience (see Table 3).

\section{Improved Mood}

Women in both arms reported improvements in their mood, a second theme not identified a priori. In the hypnosis group, these statements reflected feelings of empowerment, happiness and being outgoing. This language is consistent with that used in the hypnotic suggestions. In the PMR group, patience, energy, and emotional balance were mentioned. Participants in both groups mentioned a new willingness to share with spouse (see Table 4).

\section{Sexual Health}

The theme of sexual health (not a priori) emerged among participants in the hypnosis arm, but not the PMR arm of the study (see Table 5). This finding is consistent with the hypnotic suggestions that evolved over the six-week intervention in the hypnosis group. The first two weeks included positive suggestions about one's body and sexual needs. The middle two weeks offered suggestions about feelings of 
sexuality and the sexual experience. The final two weeks asked women to select and implement a behavior consistent with meaningful self-care.

The only quotations related to sexual health among PRM participants were in response to a question asked at the end of the intervention, "Were you satisfied with the effect this treatment had on your sexuality?" All women offering feedback in the PMR group indicated the question was not applicable.

\section{Discussion}

In our randomized phase II study, PMR was planned to be an active control but was found to have more than a placebo effect on body image stress, our primary outcome, as measured by the Impact of Treatment Scale. Our qualitative findings support that we successfully blinded our hypothesis as comments from both groups revealed engagement and positive effects on stress, relaxation, connecting the mind and body and mood.

Evidence of stress reduction, relaxation, and improved mood among the PMR group should not be surprising given effects demonstrated by other research. For example, Allison et al. [15] studied the effectiveness of PMR as a self-care strategy among first year veterinary students and found all students showed improvements in basic relaxation and stress levels after completing PMR. In a narrative review by Raad et al [16] PMR was examined along with cognitive behavioral therapy (CBT) and deep breathing (DB) among adults undergoing treatment for infertility and investigators concluded that each of these techniques was associated with improvements in mood and reduced stress levels. What is surprising is that progressive muscle relaxation could positively impact body image. Additionally surprising is that, at least in some women, these improved feelings would translate into feeling closer to their partner.

The results of this exploratory qualitative analysis led us to form some beginning hypotheses with respect to mechanisms that may improve body image. The Theory of Unpleasant Symptoms [17] states that in a person experiencing unpleasant symptoms, there is a continual and reciprocal relationship between influencing factors (physiologic, psychologic and situational), symptoms and performance. Influencing factors (such as cancer status, anxiety and decrease in intimacy with one's partner) affect a person's experience with symptoms (such as negative body image). Symptoms affect performance, or in this case, the complex status of physical, emotional, mental, and social well-being in relation to sexuality, not merely the absence of disease, dysfunction or infirmity. We propose that mechanisms through which body image may be improved include improved sleep, which decreases cortisol and can help the stress response; coping well with stress, which may improve feelings of control; increasing positive mood, which can lead to more self-care activities; and the ability to connect one's positive feelings to their physical body (mind-body connection) (see Fig. 1). Based on these qualitative findings, we propose that both Hypnosis and PMR may have improved body image through better sleep, more control over stress, positive mood and connecting the mind with the body. PMR accomplished this with overt instructions to tense and relax muscles from the top of the head down to the bottom of the feet while listening to 
pleasing nature sounds and hypnosis did this through suggestions provided to the subconscious during deep relaxation.

Several of the responses from the hypnosis group directly reflected the hypnotic suggestions used in the intervention. For example, Participant 1041 stated "more relaxation, noticeable sexual feelings, openness, interest." Relaxation, sexual feelings and interest were part of the hypnotic suggestions. Additionally the hypnosis group included findings of improved sexual health. This may be the result of the specific hypnotic suggestions around sexual health and feeling empowered. During the final weeks of the intervention period, there was evidence in the hypnosis group of increased action to make changes. For example, participant 1043 stated that she "Felt good about myself. Starting to make changes and take control over my body." This shift into action was not seen in the PMR group's responses. Similar to Jensen et al.[18] findings, where women in treatment and post treatment for breast cancer participated in self-hypnosis and an improvement was found for pain, fatigue and sleep problems, all symptoms targeted by hypnotic suggestions, we pose that the specific hypnotic suggestions made a difference that aided women to implement change in their behaviors and improve their sexual health, specifically [18]. These findings may explain the slightly higher but not statistically significant sexual satisfaction and sexual interest scores seen in the hypnosis group in the parent study [13].

One of the key limitations of this study is that participants self-selected by choosing to provide written comments on the questionnaires from the parent study. As such our findings represent women who were motivated to write comments and may not be a true representation of the entire study population. Even with this self-selection, well over half of the participants in each arm provided comments. It should also be emphasized again that comment analysis was not originally planned when this study was designed. It would have been helpful to have included a qualitative component of this study that was designed expressly for the purpose of understanding mechanisms of benefit.

Our study findings suggest that PMR and hypnosis both may improve women's stress, ability to relax, sleep, mood, and mind-body connection and these factors may result in an improved body image.

However, these comments also support the quantitative data [13] that the suggestions embedded in the hypnosis intervention did impact the women in a positive way related to sexual health. Future research should test the relationships we propose and better explore their directional and/or causal nature. Additionally, more research is needed to continue to determine which intervention (PMR or Hypnosis) is better suited to improve cancer survivors' body image and subsequently their overall sexual health.

\section{Declarations}

Funding: This study was supported by the Breast Cancer Research Foundation.

Conflicts of interest/Competing interests: The authors have no conflicts of interest to disclose.

Availability of data and material (data transparency): Not Available 
Code availability (software application or custom code): Not applicable.

Ethics approval: All procedures performed in studies involving human participants were in accordance with ethical standards of the institution and with the 1964 Helsinki declaration and its later amendments or comparable ethical standards.

Consent to participate: Informed consent was obtained from all individual participants included in the study.

Consent for publication (include appropriate statements) Informed consent included consent for publication.

\section{References}

1. American Cancer Society (2019) Cancer Treatment \& Survivorship Facts \& Figs. 2019-2021. American Cancer Society, Atlanta

2. World Health Organization (2021) Sexual Health. https://www.who.int/health-topics/sexualhealth\#tab=tab_1. Accessed May 27, 2021

3. Stabile C, Goldfarb S, Baser RE, Goldfrank DJ, Abu-Rustum NR, Barakat RR, Dickler MN, Carter J (2017) Sexual health needs and educational intervention preferences for women with cancer. Breast Cancer Res Treat 165(1):77-84. doi:10.1007/s10549-017-4305-6

4. World Health Organization (2006) Defining sexual health: Report of technical consultation on sexual health, 28-31 January 2002, Geneva. WHO Press, Geneva

5. Woertman L, van den Brink F (2012) Body image and female sexual functioning and behavior: a review. J Sex Res 49(2-3):184-211. doi:10.1080/00224499.2012.658586

6. Gillen MM, Markey $\mathrm{CH}$ (2019) A review of research linking body image and sexual well-being. Body Image 31:294-301. doi:10.1016/j.bodyim.2018.12.004

7. Ganz PA, Desmond KA, Belin TR, Meyerowitz BE, Rowland JH (1999) Predictors of Sexual Health in Women After a Breast Cancer Diagnosis. J Clin Oncol 17(8):2371-2380

8. Gilbert E, Ussher JM, Perz J (2010) Sexuality after breast cancer: a review. Maturitas 66(4):397-407. doi:10.1016/j.maturitas.2010.03.027

9. Lewis-Smith H, Diedrichs PC, Rumsey N, Harcourt D (2018) Efficacy of psychosocial and physical activity-based interventions to improve body image among women treated for breast cancer: $A$ systematic review. Psychooncology 27(12):2687-2699. doi:10.1002/pon.4870

10. Raggio GA, Butryn ML, Arigo D, Mikorski R, Palmer SC (2014) Prevalence and correlates of sexual morbidity in long-term breast cancer survivors. Psychol Health 29(6):632-650. doi:10.1080/08870446.2013.879136

11. Abbott-Anderson K, Young PK, Eggenberger SK (2020) Adjusting to sex and intimacy: Gynecological cancer survivors share about their partner relationships. J Women Aging 32(3):329-348. 
doi:10.1080/08952841.2019.1591888

12. Elkins GR, Barabasz AF, Council JR, Spiegel D (2015) Advancing Research and Practice: The Revised APA Division 30 Definition of Hypnosis. Am J Clin Hypn 57(4):378-385.

doi:10.1080/00029157.2015.1011465

13. Barton DL, Brooks TM, Cieslak A, Elkins GR, Clark PM, Baydoun M, Smith AB, Van Poznak CH (2019) Phase II randomized controlled trial of hypnosis versus progressive muscle relaxation for body image after breast or gynecologic cancer. Breast Cancer Res Treat 178(2):357-365. doi:10.1007/s10549-019-05395-6

14. Fereday J, Muir-Cochrane E (2006) Demonstrating Rigor Using Thematic Analysis: A Hybrid Approach of Inductive and Deductive Coding and Theme Development. International Journal of Qualitative Methods 5 (1)

15. Allison S, Hamilton KI, Yuan Y, Hague GW (2019) Assessment of Progressive Muscle Relaxation (PMR) as a Stress-Reducing Technique for First-Year Veterinary Students. J Vet Med Educ:e20180013. doi:10.3138/jvme.2018-0013

16. Raad GT, Azoury J, Daher J, FAkih A, Bakos C, Hassan W (2020) Neurophysiology of cognitive behavioral therapy, deep breathing and progressive muscle relaxation used in conjunction with ART treatments: A narrative review. Human Reproduction Update:1-15

17. Lenz E, Pugh LC, Milligan RA, Gift A, Suppe F (1997) The middle-range theory of unpleasant symptoms: An update. Adv Nurs Sci 19(3):14-27

18. Jensen MP, Gralow JR, Braden A, Gertz KJ, Fann JR, Syrjala KL (2012) Hypnosis for symptom management in women with breast cancer: a pilot study. Int J Clin Exp Hypn 60(2):135-159. doi:10.1080/00207144.2012.648057

\section{Tables}

Table 1

Select quotations illustrating the theme relaxation and stress management

\section{Hypnosis Group}

(I felt) relief from anxiety after practice, positive feelings - hopeful and calm - about participating in the study. (Week 1, Participant 1041).

(I felt) more peace (Week 1, Participant 1054).

Reached very relaxed state. (Week 3, Participant 1027).

Saw colors, relaxed deeply (Week 4, Participant 1008).

\section{PMR group}

(It has been an) extraordinarily stressful week. Grateful to have a focus to try to quell some of the anxiety (Week 2, Participant 1070).

Felt so good went through it twice (Week 4, Participant 1083).

Helpful to reduce stress (Week 5, Participant 1068).

Afterwards I felt really relaxed (Week 6, Participant 1032). 
Table 2

Select quotations illustrating the theme sleep

Hypnosis Group

Noticed I drifted but opened my eyes immediately when the \#1 was spoken (awakening cue in hypnosis) (Week 2, Participant 1008).

Slept the best I have in months (Week 2). Relaxed, slept great (Week 5, Participant 1026).

Slept better (Week 2). Better sleep (Week 3). Great sleep (Week 4, Participant 1046).

Felt myself have several falling asleep spasms even though I felt fully awake (Week 3, Participant 1022).
PMR group

Almost fell asleep (Week 1 , Participant 1008, Week 2, Participant 1073)

I slept better on all but one night (Week 1, Participant 1083).

Fell asleep (Week 3, Participant 1083 and Week 5, Participant 1055)

Kept falling asleep (Week 6, Participant 1028).

Table 3

Select quotations illustrating the theme mind-body connection

\section{Hypnosis Group}

Felt good about myself. Starting to make changes and take control over my body. (Week 4, Participant 1043).

People told me I looked and acted younger, slimmer. Nice! (Week 4, Participant 1046)

\section{PMR group}

Energized, soothed, invigorated, confident, strong (Week 1, Participant 1048).

Some muscle pain improvement and I feel more aware of all parts of me. (Week 6, Participant 1078)

Very motivated to engage in better self-care (Week 3). Consistently exercise, slept and ate better than usual (Week 4, Participant 1095).

More confident in my workouts; less stressed, more focused. (Week 6, Participant 1015) 
Table 4

Select quotations illustrating the theme improved mood

Hypnosis Group

(I feel) a sense of well-being/happiness, strong on day of meeting (Week 1, Participant 1057).

(I) started to make changes in my life. Looking to make myself happier (Week 3, Participant 1043).

Empowered/back on track (Week 5, Participant 1054).

One of my goals seemed unrelated to sexual or self-image resuming my learning to paint - but it made me feel incredibly satisfied and accomplished to do it. The other goal - improved communication with my husband - seems to make us both happier. (Week 6, Participant 1089).

(I feel) more enjoyment in life (Week 6, Participant 1045).

\section{PMR group}

More patience than usual (Week 1 , Participant 1012).

Closer to husband after discussion of issues (Week 1, Participant 1021).

(Practiced at...) sunrise early morning. Place full of energy rest of day (Week 3, Participant 1073).

Much more aware of my lack of willingness to be vulnerable (Week $3)$. Crying, sharing more openly $\mathrm{w} / \mathrm{my}$ husband about feelings (Week 4, Participant 1070).

I have felt more calm and balanced emotionally. (Week 6, Participant 1083).

Table 5

Select quotations illustrating the theme sexual health

\section{Hypnosis Group}

(I feel) more relaxation, noticeable sexual feelings, openness, interest. (Week 4). I am optimistic that the more I practice/listen to recordings, my feelings about my sexuality will continue to improve (Week 6, Participant 1041).
PMR group

No comments addressed changes in sexual health specifically.

(I am) feeling more sexual - having fun (Week 5, Participant 1045).

\section{Figures}



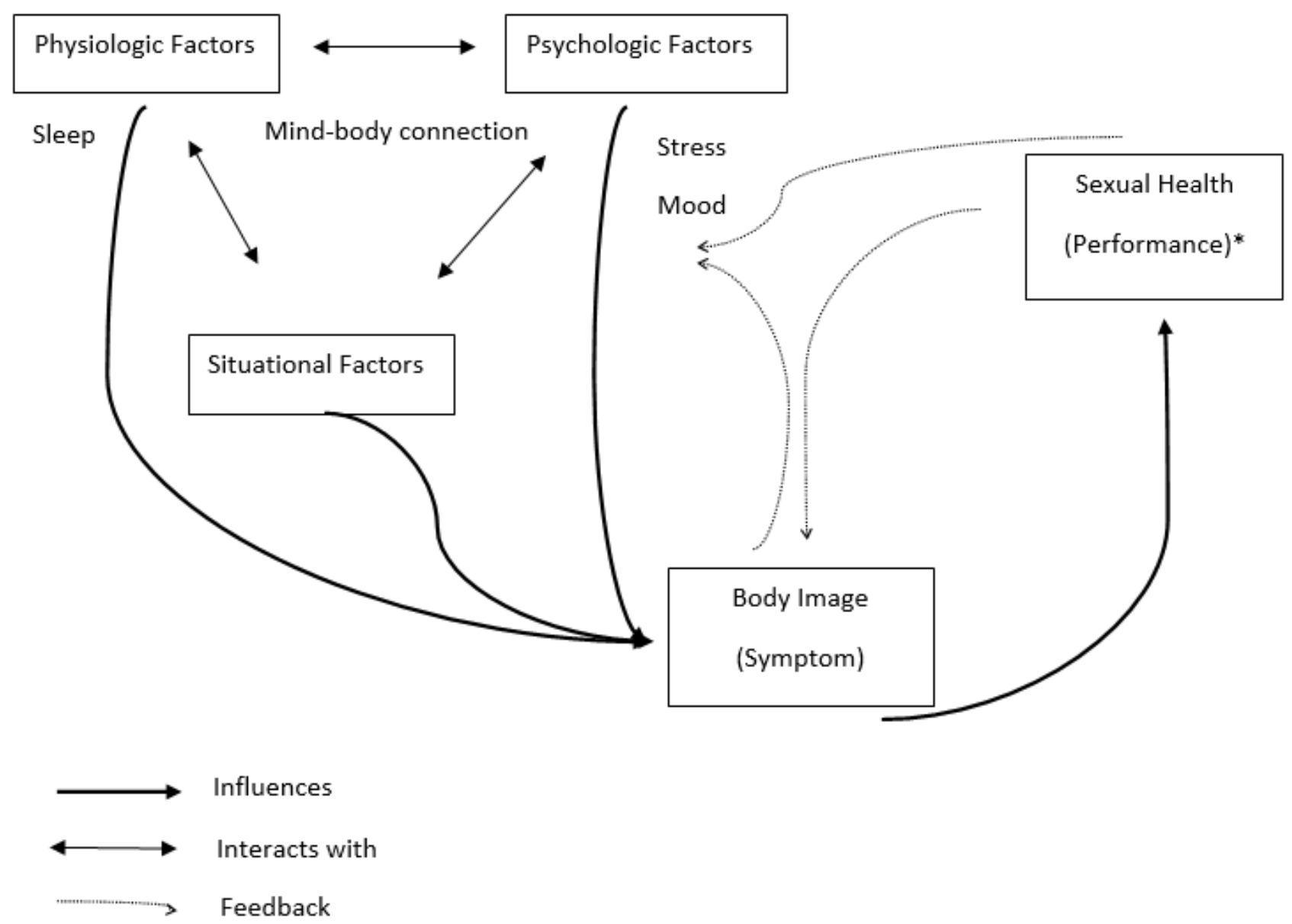

Figure 1

Proposed mechanisms for improved body image. Adapted from the middle-range theory of unpleasant symptoms: An update [1]. A continuing and reciprocal relationship occurs between influencing factors (sleep, mind-body connection, stress and mood), symptoms (body image) and performance (a complex status of sexual health). The influences and interactions illustrate how both PMR and self-hypnosis might have contributed to improved body image. 